\title{
ORIGINAL
}

\section{VIGILANCIA DE LA GRIPE PANDÉMICA EN LA COMUNIDAD VALENCIANA Y VACUNA ANTIGRIPAL ESTACIONAL}

\author{
Rosa María Carbó Malonda, Francisco González Morán, Hermelinda Vanaclocha Luna, Miguel \\ Martín-Sierra Balibrea, Silvia Guiral Rodrigo, Elvira Pérez Pérez, Teresa Castellanos Martínez, \\ del Grupo de trabajo de Vigilancia Epidemiológica de La Comunitat Valenciana
}

Área de Epidemiología; Dirección General de Salud Pública; Consellería de Sanitat de la Comunitat Valenciana.

\section{RESUMEN}

Fundamento: En la Comunidad Valenciana se ha llevado a cabo la vigilancia de la Gripe pandémica. Algunos estudios sobre efectividad de la vacuna estacional para Gripe AnH1N1 han presentado resultados no consistentes. El objetivo del trabajo es describir los resultados de la vigilancia epidemiológica y la efectividad de la vacuna estacional para Gripe pandémica en las semanas 28 a 51 de 2009.

Métodos: Se estudiaron los casos en atención primaria, hospitalizados confirmados, aislamientos virales y reacción de la cadena de la polimerasa (PCR) y coberturas vacunales. Se calculó la efectividad vacunal por el método de screening de Farrington, en tres grupos de edad y dos periodos: vacunados de las temporadas 2008-9 y 2009-10.

Resultados: En el primer periodo (semanas 28 a 40) los casos se concentraron en el grupo de 15 a 64 años ( 7.207 casos), seguido de los menores de 15 años (1.596 casos). En el segundo periodo (semanas 45 a 47) afectó más a menores de 15 años (28.218 casos). En ambos periodos las tasas de incidencia en mayores de 65 años fue de 56,3 y 125,1 respectivamente. En el periodo estudiado (semanas 28 a 51) se confirmaron 5.481 casos de los que 1.746 (31,8\%) fueron hospitalizados. La curva de personas hospitalizadas presentaba un perfil similar al de atención primaria, y también el seguimiento microbiológico del virus. La efectividad vacunal en el segundo periodo fue del $25 \%$ en adultos entre 15 y 64 años y del $51 \%$ en mayores de 64 años.

Conclusiones: Se observó una protección edad dependiente con efectividad vacunal positiva en los mayores de 64 años, aunque puede estar confundida por exposición natural al virus, vacunas previas y o respuesta inmunitaria.

Palabras clave: Brote. Pandemia. Subtipo H1N1 del Virus de la Influenza A. Gripe humana. España. Vacunas contra la Influenza. Efectividad.

\section{ABSTRACT}

Background: Surveillance of Pandemic influenza was carried out in the Valencian Community. Some effectiveness studies of the seasonal vaccine for AnH1N1 virus have presented no consistent results. The objective of the work consists on describing the results of the epidemic surveillance and effectiveness of the seasonal vaccine for pandemic influenza in the weeks 28 to $51,2009$.

Methods: We studied the cases in primary care, hospitalized confirmed, Polimerase Chain Reaction (PCR) and viral isolates and vaccine coverage. Vaccine effectiveness was calculated by the Farrington method of screening method, in three age groups, and two periods: vaccinated 2008-9 and 2009-10 seasons.

Results: In the first period (weeks 28-40) the incidence rates were highest in the group of 15-64 years (7207 cases), followed by those under 15 years (1596 cases). In the second period (weeks $45-$ 47 ) children under 15 years ( 28218 cases) were the most affected. In both periods incidence in patients older than 65 years was low (rates of 56,3 and 125,1 respectively). In the studied period (weeks 28 at 51) 5481 cases were confirmed, of those that $1746(31,8 \%)$ were hospitalized. The curve of hospitalization rate showed a profile similar to those in primary care and also in microbiological surveillance of the virus. The vaccine effectiveness in the second period was $25 \%$ in adults between 15 and 64 years and $51 \%$ in those older than 64 years.

Conclusions: There is an age-dependent protection with positive vaccine efficacy in the elderly, although it may be confounded by natural exposure to the virus, previous immunizations or immune response.

Key words: Disease Outbreaks. Influenza A virus, H1N1 subtype. Grippe. Influenza, human. Spain. Influenza virus vaccines. Effectiveness. Pandemic

Correspondencia:

Francisco González Morán.

Avenida de Cataluña, 21 CP 46021 Valencia

Correo electrónico: gonzalez_framor@gva.es 


\section{INTRODUCCIÓN}

El 11 de Julio de 2009 la Organización Mundial de la Salud (OMS) declaró la fase 6 de pandemia por la nueva gripe (H1N1) $2009^{1,2}$. El nuevo virus emergió de una reorganización genética de diversos linajes de virus de la gripe porcina ${ }^{3}$. El primer caso en la Comunidad Valenciana ocurrió en la semana 17 del año 2009 (26 de abril a 1 de mayo) y desde entonces se llevó a cabo la vigilancia a través del Sistema de Análisis de la Vigilancia Epidemiológica (AVE), en tiempo real, con la declaración individual de todos los casos con sospecha clínica de Gripe, atendidos en la red pública de la Comunidad Valenciana. Este sistema, además, permite el cruce con el Sistema de Información Vacunal (SIV) para conocer la situación vacunal del individuo frente a la gripe estacional. Recientemente se han publicado diferentes estudios sobre la forma de presentación y dinámica de la pandemia ${ }^{4,5}$. El virus pandémico circulante en el hemisferio norte era antigénica y genéticamente similar al A/California/7/20096,7,8. Algunos estudios sobre la efectividad de la vacuna de Gripe estacional para la prevención de la Gripe (H1N1) 2009, de manera complementaria a los estudios serológicos, han presentado resultados no consistentes ${ }^{9,10-13}$.

El objetivo de este trabajo es presentar los resultados del sistema de vigilancia de la Gripe entre la semana 28 y la 51 del año 2009 a partir del sistema informático AVE y estimar la efectividad que la vacuna de la Gripe estacional ha tenido para la prevención de la Gripe pandémica en la Comunidad Valenciana.

\section{SUJETOS Y MÉTODOS}

Se han utilizado las siguientes fuentes de datos:

- Atención Primaria: casos diagnosticados y declarados por el médico de familia o pediatra y registrados en la historia clínica electrónica como síndrome gripal (Influenza Like Illness: ILI), sin identificación viral.

- Hospital: casos confirmados a Gripe (H1N1) 2009 entre los que se distinguen los hospitalizados no graves, graves, graves en Unidad de Cuidados Intensivos (UCI), y los fallecidos. Se consideran casos graves las gripes confirmadas a virus pandémico, ingresadas en UCI o en planta, y que presentan neumonía y/o fallo multiorgánico y/o shock séptico.

- La Red Microbiológica Valenciana (RedMIVA) complementa el sistema AVE aportando la información de los aislamientos virales y PCR frente a Gripe (H1N1) 2009 para cada uno de los casos hospitalizados.

- El SIV es una fuente de información integrada que permite conocer el estado vacunal de cada uno de los casos notificados y las coberturas vacunales de la población general desagregada por grupos de edad.

Se han estudiado las variables de edad, grado de confirmación, hospitalización, gravedad y estado vacunal con gripe estacional. Se ha dispuesto también de la cobertura vacunal poblacional por grupos de edad y del grado de confirmación de laboratorio para el cálculo del Valor Predictivo Positivo (VPP).

Los periodos estudiados fueron:

- Semanas 28 a 40 (de 12 de julio a 10 de octubre), para el análisis de la vacuna de la temporada 2008-2009 a partir de la cual se inicia la campaña de vacunación de la siguiente temporada.

- Semanas 45, 46 y 47 (de 8 a 28 de noviembre), para el análisis de la vacu- 
na de la temporada 2009-2010, cuando la mayoría de los vacunados habían recibido la vacuna al menos dos semanas antes.

La vacuna estacional de la temporada 2008-09 contenía las siguientes cepas: Cepa análoga a A/Brisbane/59/2007 (H1N1); Cepa análoga a A/Brisbane/10/2007 (H3N2) y Cepa análoga a B/Florida/4/2006. En la siguiente temporada únicamente se sustituyó la cepa B/Florida/4/2006 por la B/Brisbane/60/2008 (linaje Victoria).

A partir de estos datos se ha calculado la efectividad de las vacunas de gripe estacional de las temporadas 2008-2009 y 20092010 de forma independiente, utilizando la definición de caso en Atención Primaria, mediante un diseño ecológico caso-cohorte.

Para el cálculo de la efectividad vacunal se ha utilizado el método de screening de
Farrington a partir de los datos de vacunación de los casos y de la proporción de población vacunada global y por grupos de edad en tres estratos, como método exploratorio, y se ha calculado después por el método directo de Orenstein ${ }^{17,18}$.

\section{RESULTADOS}

La incidencia de gripe en atención primaria desde la semana 28 hasta la semana 43 pasó de una tasa de 2,7 casos por $10^{5}$ habitantes a 70,2. En la semana 43 comenzó la onda epidémica, caracterizada por un ascenso y una bajada bruscas, llegando a 506 casos por $10^{5}$ habitantes en la semana 46 , con tasas superiores en el grupo de 5 a 14 años (2090 casos por $10^{5}$ niños) e inferiores en los mayores de 64 años (figuras 1 y 2).

En el primer periodo (semanas 28 a 43) los casos declarados se concentraron en el

Figura 1

Tasas semanales de Gripe pandémica por grupos de edad en Atención Primaria

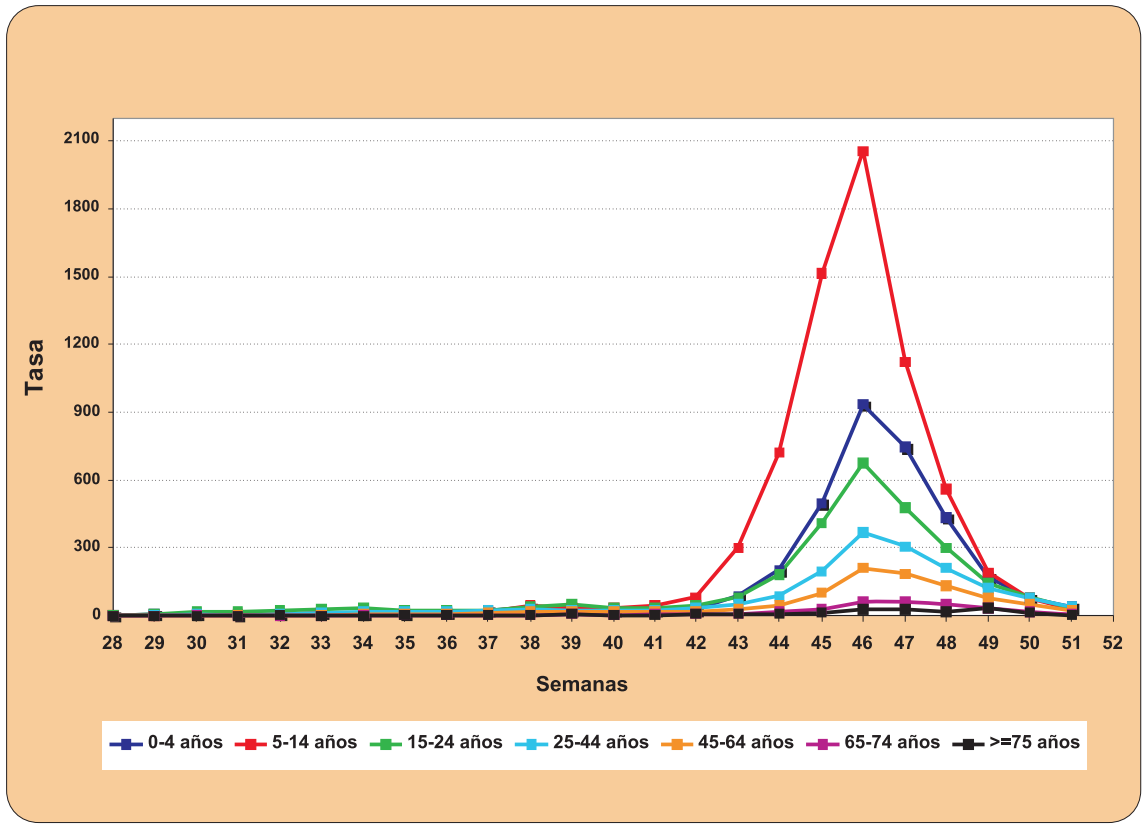


Figura 2

Tasas semanales de Gripe pandémica y casos hospitalizados

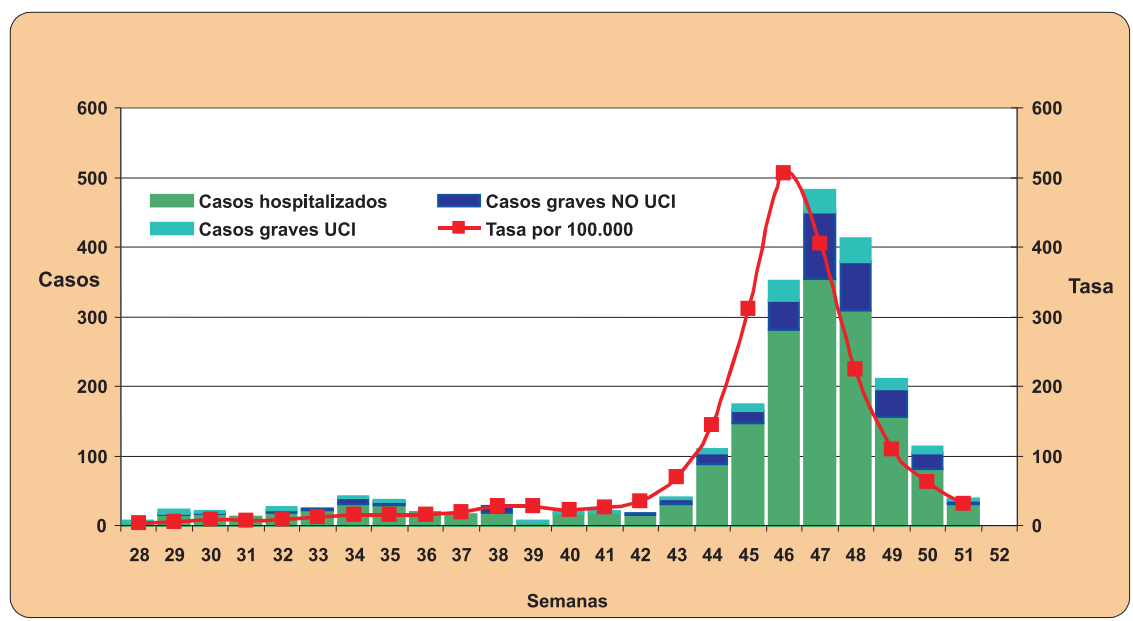

Figura 3

Prevalencia de enfermedades de base en los casos graves de gripe pandémica



grupo de edad de 15 a 64 años (10.837 casos), seguido de los menores de 15 años (3952 casos), con muy baja incidencia en el grupo de mayores de 65 años (668 casos). En el segundo periodo (semanas 44 a 51) se observó un incremento próximo a diez veces en la declaración de casos en menores de 15 años (37.132 casos), siendo este incremento menos notorio en el resto de grupos de edad. 
Tabla 1

Indicadores de evolución de la Gripe pandémica en la Comunitat Valenciana en las semanas 28 a 51 (12 de julio al 26 de diciembre) de 2009

\begin{tabular}{|l|c|}
\hline \multicolumn{1}{|c|}{ INDICADORES } & RESULTADO \\
\hline Total casos notificados de síndrome gripal por Atención Primaria & 104.141 \\
\hline Casos confirmados por laboratorio & 5.481 \\
\hline Casos hospitalizados confirmados & 1.746 \\
\hline Casos en UCI & 155 \\
\hline Casos fallecidos & 35 \\
\hline Tasa de hospitalizaciones por 100 enfermos & $1,67 \%$ \\
\hline Tasa de UCI por 100 hospitalizados & $8,9 \%$ \\
\hline Tasa de letalidad por 100 enfermos & $0,034 \%$ \\
\hline Tasa de ataque clínica por 100 habitantes & $2,13 \%$ \\
\hline
\end{tabular}

La curva de los casos hospitalizados por gripe pandémica tuvo un perfil similar a la de los casos de gripe declarados en Atención Primaria, aunque con un retardo de una semana (figura 2); la mayor parte de los casos graves se dieron en los grupos de edad de 25 a 64 años, que supusieron el 55,2\% (288/522) del total de los casos graves. No existieron factores de riesgo conocido en el $23,2 \%(36 / 155)$ de los sujetos ingresados en UCI, y tampoco en el 37,6\% (138/367) de los ingresados en salas generales. Las enfermedades de base mas frecuentes en los 155 casos graves que ingresaron en UCI fueron la obesidad (13,9\%), la enfermedad pulmonar $(12,6 \%)$ y la cardiopatía $(12,6 \%)$, y entre los 367 ingresados en sala general destaca la diabetes $(11,6 \%)$ y la enfermedad pulmonar $(11,3 \%)$ (figura 3$)$.

Como indicadores de evolución de la gripe pandémica hay que destacar que durante el período de seguimiento la tasa de ataque clínica fue del 2,1\% habitantes, la tasa de hospitalización del 1,7\% de los enfermos, la tasa de UCI del 9,5\% de los hospitalizados y la letalidad del 34 por $10^{5}$ enfermos (tabla 1).

Vigilancia de la gripe pandémica a través de la RedMIVA.- El seguimiento microbiológico del virus (H1N1) 2009 mostró una incidencia estabilizada desde la semana 28 hasta la semana 42 (tasas que oscilaron entre 0,7 y 1,9 casos por $10^{5}$ ), a partir de la cual aparece una curva similar a la de los casos declarados por Atención Primaria. El resto de virus gripales tuvieron una mínima representación en la mencionada curva; no obstante, las tres últimas semanas (49 a 51) se detectó el Virus Respiratorio Sincitial (VRS), que se incrementó de forma progresiva hasta superar en la semana 51 al virus pandémico (78 aislamientos de VRS frente a 49 Virus pandémico) (figura 4).

Para comparar el valor del diagnóstico clínico se calculó el VPP en ambos periodos. El VPP fue muy bajo en el primero, siendo en el grupo de mayores de 65 años solamente de un $6 \%$. En el segundo periodo aumentó considerablemente y alcanzó el $71 \%$ en los 
Figura 4

Virus de la gripe pandémica y otros virus respiratorios identificados por la Redmiva en las semanas 28 a 51

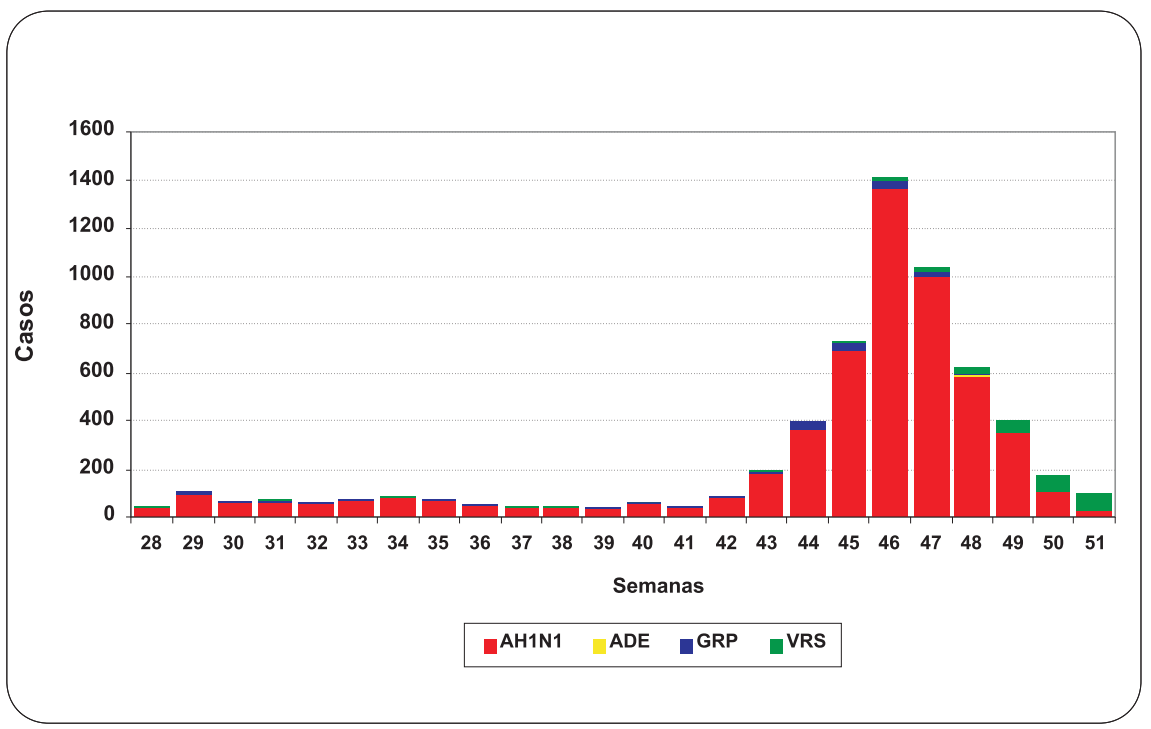

menores de 15 años y en los mayores de 65 años se alcanzó el $29 \%$. En este periodo los criterios de recogida de muestras habían variado y prácticamente todas las muestras provenían de los casos hospitalizados.

Coberturas de la vacuna estacional en la temporada 2009-2010 por grupos de edad.En las cuatro primeras semanas de la campaña de vacunación antigripal estacional (semanas 40 a 43) se pusieron el $89 \%$ de la totalidad de las vacunas de la temporada 2009-2010, de modo que al final de la semana 43, que finalizó el 31 de octubre, se habían alcanzado coberturas acumuladas próximas a la cobertura final, que en los menores de 15 años fue del 4,7\% (38.258 dosis), en el grupo de 15 a 64 años del 7,8\% (278.454 dosis), y en los mayores de 64 años del $56,1 \%$ (482.152 dosis).

Efectividad de la vacuna estacional. Entre la semana 28 y la 40 se declararon al sistema AVE 9250 casos (tasa de 189,3) de gripe clí- nica, de los cuales 1311 (14,2\%) estaban vacunados con la vacuna estacional de 20082009. La incidencia más elevada se dio en los menores de 15 años (tasa de 225,5 casos por $10^{5}$ habitantes). Cuando se estudian las tasas según el antecedente de vacunación, se observa que en los no vacunados (tasa de 191,2) ésta es ligeramente mas elevada que en los vacunados (tasa de 179,1); sin embargo, al estructurarse por grupos de edad se produce un cambio en las tasas pasando a ser mas elevadas en los tres grupos de edad para los vacunados (tabla 2 ).

En las semanas 45, 46 y 47, una vez finalizada la campaña de vacunación de la gripe estacional de la temporada 2009-2010, hay un incremento de la incidencia de la enfermedad con una declaración de 59644 casos (tasa de 1166,7) especialmente en niños menores de 15 años (tasa de 3610,5). En este periodo de tres semanas el riesgo de enfermar en los niños vacunados es superior al de los no vacunados (RR: 1,19; IC95\% 
Tabla 2

Casos de Gripe pandémica declarados por Atención Primaria en las semanas 28 a 40 (12 de julio al 10 de octubre) del año 2009,

Riesgo relativo y Efectividad de la vacuna antigripal estacional 2008-2009

\begin{tabular}{|c|c|c|c|c|c|c|c|c|}
\hline \multirow[b]{2}{*}{ Edad } & \multicolumn{3}{|c|}{ VACUNADOS } & \multicolumn{3}{|c|}{ NO VACUNADOS } & \multirow{2}{*}{$\begin{array}{c}\mathbf{R R}^{*} \\
\text { Vacunado/No } \\
\text { Vacunado }\end{array}$} & \multirow{2}{*}{$\begin{array}{l}\text { Efectividad } \\
\text { Método } \\
\text { Directo }\end{array}$} \\
\hline & Población & Casos & $\begin{array}{c}\text { Tasa } \\
\text { por } 10^{5}\end{array}$ & Población & Casos & $\begin{array}{c}\text { Tasa } \\
\text { por } 10^{5}\end{array}$ & & \\
\hline$<15$ & 21.510 & 105 & 488 & 686.067 & 1.491 & 217 & $\begin{array}{c}2,25 \\
(1,8 ; 2,7)\end{array}$ & $\begin{array}{c}-1,2 \\
(-1,7 ;-0,84)\end{array}$ \\
\hline $15-64$ & 252.593 & 922 & 365 & 3.130 .942 & 6.285 & 200 & $\begin{array}{c}1,81 \\
(1,7 ; 1,9)\end{array}$ & $\begin{array}{c}-0,8 \\
(-0,9 ;-0,7)\end{array}$ \\
\hline$>=65$ & 457.931 & 284 & 62 & 335.986 & 163 & 48 & $\begin{array}{c}1,27 \\
(1,05 ; 1,55)\end{array}$ & $\begin{array}{c}-0,27 \\
(-0,5 ;-0,05)\end{array}$ \\
\hline
\end{tabular}

$* \mathrm{RR}=$ Riesgo relativo

Tabla 3

Casos de Gripe pandémica declarados por Atención Primaria en las semanas 45 a 47 (8 al 28 de noviembre) del año 2009, Riesgo relativo y efectividad de la vacuna antigripal estacional 2009-2010

\begin{tabular}{|c|c|c|c|c|c|c|c|c|}
\hline \multirow[b]{2}{*}{ Edad } & \multicolumn{3}{|c|}{ VACUNADOS } & \multicolumn{3}{|c|}{ NO VACUNADOS } & \multirow{2}{*}{$\begin{array}{c}\mathbf{R R}^{*} \\
\text { Vacunado/No } \\
\text { Vacunado }\end{array}$} & \multirow{2}{*}{$\begin{array}{c}\text { Efectividad } \\
\text { Método } \\
\text { Directo }\end{array}$} \\
\hline & Población & Casos & $\begin{array}{c}\text { Tasa } \\
\text { por } 10^{5}\end{array}$ & Población & Casos & $\begin{array}{c}\text { Tasa } \\
\text { por } 10^{5}\end{array}$ & & \\
\hline$<15$ & 28.739 & 1.230 & 4.279 & 752.801 & 26.988 & 3.583 & $\begin{array}{c}1,19 \\
(1,12 ; 1,26)\end{array}$ & $\begin{array}{c}-0,19 \\
(-0,26 ;-0,12)\end{array}$ \\
\hline $15-64$ & 249.731 & 1.653 & 661 & 3.240 .868 & 28.780 & 888 & $\begin{array}{c}0,75 \\
(0,7 ; 0,78)\end{array}$ & $\begin{array}{c}0,25 \\
(0,21 ; 0,29)\end{array}$ \\
\hline$>=65$ & 456.564 & 362 & 79 & 383.636 & 631 & 164 & $\begin{array}{c}0,48 \\
(0,42 ; 0,54)\end{array}$ & $\begin{array}{c}0,51 \\
(0,45 ; 0,57)\end{array}$ \\
\hline
\end{tabular}

$* \mathrm{RR}=$ Riesgo relativo

1,12-1,26); sin embargo el riesgo disminuye de forma progresiva en el adulto joven vacunado (RR: 0,75 ; IC 95\% 0,7-0,78) y en los mayores de 64 años (RR: 0,48; IC 95\% $0,42-0,54)$, equivalentes a una efectividad de la vacuna del 25\% (IC95\% 20-30\%) en los sujetos entre 15 y 64 años y del $51 \%$ (IC95\% 45-57\%) en los mayores de 64 años (tabla 3).

\section{DISCUSIÓN}

En general nuestros resultados son consistentes con otros sistemas de vigilancia epidemiológica españoles en cuanto a la incidencia, distribución y dinámica de la Gripe pandémica, siendo el grupo de edad más afectado el de menores de 15 años, similar al reportado en algunas áreas del hemisferio 
sur $^{4}$ y caracterizándose el comportamiento del virus por su rápida difusión y baja letali$\mathrm{dad}^{5}$.

La definición de caso de Gripe utilizada se ha considerado válida por su alta sensibilidad, aún sin ser confirmada por el laboratorio, existiendo un paralelismo entre la curva de casos de Gripe clínica en Atención primaria y la de los hospitalizados y confirmados. En contra de esta estimación estaría el bajo VPP del diagnóstico clínico especialmente en los mayores de 65 años, en ambos periodos estudiados, pero de una magnitud diferente en cada uno de ellos. El VPP presenta la limitación de depender de la incidencia de la enfermedad a diagnosticar; así durante el primer periodo la incidencia fue muy baja en los mayores de 65 años y un diagnóstico clínico no permitiría la confirmación y podría dar lugar a un sesgo de mala clasificación de la enfermedad. En cambio, en el segundo periodo cuando la incidencia fue mayor un diagnóstico clínico tendería a confirmar la presencia de la enfermedad lo que reduciría el sesgo de mala clasificación, que además se estaría aplicando tanto a vacunados como a no vacunados, por lo que el sesgo cometido seria no diferencial y no afectaría al resultado final del cálculo de la efectividad. Por otra parte, el $95 \%$ de los virus respiratorios identificados en la Comunidad Valenciana hasta la semana 51 ha correspondido al virus gripal (H1N1) 2009, lo que descartaría la etiología por otros virus gripales.

Con respecto al sesgo de declaración es poco probable ya que en nuestro caso analizamos los casos declarados en toda la población de la Comunidad Valenciana, y afectaría tanto a vacunados como a no vacunados, pero de diferente manera según la edad. Por otra parte, el sesgo de prescripción iría en contra del resultado obtenido, ya que los sujetos más enfermos o más consumidores de los servicios sanitarios, que serían los de mayor probabilidad de acudir al médico en caso de gripe, serían además los grupos más vacunados.
El diseño parcialmente ecológico casocohorte ha sido utilizado en otros estudios de efectividad vacunal ${ }^{10}$. Este tipo de diseño podría ser válido cuando la enfermedad es poco frecuente y la cohorte fija, condiciones que confluyen en el presente estudio, aunque podría afectarse por el sesgo ecológico al no presentar los resultados estratificados por posibles factores de confusión o modificadores de efecto no tenidos en cuenta con los datos disponibles, como el estado inmunitario previo, la presencia de enfermedad de base, tratamientos con antivirales y otros fármacos, etc.

En cuanto a la vacuna de la Gripe estacional, está recomendada en nuestra Comunidad autónoma a diferentes grupos de riesgo, en función de la presencia de enfermedades de base previas, pero también a determinados grupos de población sin enfermedades de base conocidas como cuidadores, sanitarios, personal de servicios esenciales y mayores de 60 años. Los resultados obtenidos podrían presentar cierto grado de confusión debido a que las coberturas vacunales muestran gran variación entre los grupos de edad y es posible que los factores de riesgo para la indicación de la vacuna se distribuyan de diferente manera; así en el grupo de los menores de 15 se habría limitado la vacunación a personas con antecedentes de riesgo.

Por otra parte, cabe la posibilidad del diferente efecto de las cepas de las vacunas estacionales de ambos periodos y del número de dosis administradas; los mayores tendrían dos dosis de la cepa A (H1N1) estacional, en cambio la mayoría de los menores de 15 años solo tendrían una dosis; por tanto el número de dosis de vacuna estacional seria otra variable a ajustar y podría resultar en un posible efecto dosis-respuesta, contribuyendo a la mayor efectividad en el grupo de mayores ${ }^{16}$. A pesar de ser dos vacunas iguales, en los diferentes periodos, en lo que respecta a la cepa A (H1N1), son antigénicamente distintas de la vacuna de la gripe pan- 
démica 2009. En los estudios de efectividad de la vacuna gripal, cuando la cepa circulante no se corresponde antigénicamente con la incluida en la vacuna, la efectividad cae, si bien siempre mantiene cierta protección. ${ }^{7}$ Por tanto, no es de extrañar que en los estudios del $\mathrm{CDC}^{8}$ analizando la neutralización del virus A/California/7/2009 (cepa pandémica) con pares de sueros de ensayos clínicos con diversas vacunas antigripales estacionales (parenteral, intranasal y cepas de diversos años), no mostraran protección significativa. No obstante y dada la relatividad de los análisis serológicos como correlato de protección, se hace necesario estudios clínico-epidemiológicos. En este respecto se han realizado tres estudios con resultados discrepantes, así García y García ${ }^{9}$ en un análisis de casos y de controles encuentran una protección de la vacuna estacional utilizada en el hemisferio sur en 2009 del 73\% (IC95\%: 34$89 \%$ ) para evitar hospitalizaciones con gripe confirmada, mientras que en el estudio de casos-cohortes realizado por los CDC de los Estados Unidos ${ }^{10}$ no encuentran el mismo resultado, como tampoco lo hace un análisis de la red centinela de Australia analizando casos de gripe confirmada y atendida en atención primaria con la vacuna 2008$2009^{11}$. La población estudiada por García y García ${ }^{9}$ presenta características que podrían apuntar hacia un sesgo de selección importante ${ }^{10}$, ya que los controles de este estudio se seleccionaron en un hospital para enfermos respiratorios donde habría una mayor proporción de vacunados por su patología de base comparado con la población general. Asimismo el estudio de los $\mathrm{CDC}^{8}$ puede tener limitaciones como el escaso ajuste por variables de confusión, y sesgos de recuerdo en lo relativo a la situación vacunal. En el estudio Australiano de Kelly y Grant ${ }^{11}$, no encuentran casos de gripe confirmada en el grupo de edad de mayores de 64 años, donde en nuestro estudio la vacuna es más efectiva.

En cuanto a los antecedentes de exposición a virus de la Gripe el sistema inmunitario de los mayores ha tenido a lo largo de su vida mayor probabilidad de contacto con otras cepas de virus gripal que pueden haber provocado la producción de anticuerpos protectores de reacción cruzada de manera natural. ${ }^{9}$ En un estudio reciente llevado a cabo en Finlandia, con sueros recogidos en 2004 y 2005 describe la presencia de anticuerpos de protección cruzada preexistentes para el virus pandémico y probablemente originados por el influenza virus español y sus inmediatos descendientes ${ }^{17,18}$.

Otra limitación resulta de la comparación de los resultados obtenidos en el cálculo de la efectividad vacunal en ambos periodos, que podría explicarse por la diferencia del número de casos debido a la dinámica de la enfermedad y la diferente actuación para su control. Se podría producir un sesgo de mala clasificación de la exposición en el segundo periodo por no tener en cuenta los casos asintomáticos cuya inmunidad natural se adquiere por contacto con enfermo, cuando el número de casos en la población es alto, y ocurren formas asintomáticas o paucisintomáticas no diagnosticadas con un papel transmisor importante. Además, las medidas de control adoptadas fueron diferentes en cada uno de los periodos, desde medidas de contención en el primero, con lo que la probabilidad de exposición al virus es menor, frente a estrategias de mitigación en el segundo con una probabilidad de infectarse mayor, aumentando también la inmunidad natural de grupo, independiente del efecto de la vacuna estacional. Pero este sesgo de haberse producido seria no diferencial y afectaría tanto a vacunados como a no vacunados y a todos los grupos de edad.

La mayor efectividad observada en el grupo de mayores, por tanto, puede estar confundida por diferentes factores, relacionados con los antecedentes de exposición natural o debida a la vacuna y respuesta inmunitaria que podrían presentar un efecto de colinealidad. Algunos estudios sugieren que la respuesta a las vacunas gripales dependería de los diferentes antígenos y la respuesta inmu- 
nitaria celular y serológica, que además presentaría un diferente resultado si se estratifica en intervalos etarios más precisos, especialmente en los más mayores ${ }^{19}$.

Nuestros resultados apuntan hacia una protección edad dependiente para la gripe pandémica, y probablemente relacionada con un efecto dosis-respuesta. Por tanto y con el fin de confirmar la hipótesis generada en nuestro estudio, sería conveniente el abordaje mediante un estudio analítico ajustado de los casos.

\section{AGRADECIMIENTOS}

El grupo de trabajo de vigilancia epidemiológica de La Comunitat Valenciana está formado por Adell Aparicio Carmen, Alfonso Sánchez José Luis, Almela García Rafael, Amador Proas Concepción, Amat Vidal Miguel Ángel, Amorós Cantero Aurora, Aranaz Andrés Jesús María, Arencibia Jiménez Mercedes, Arnedo Pena Alberto, Barrenengoa Sañudo Julio, Bautista Rentero Daniel, Bayo Gimeno Juan, Bellido Blasco Juan, Beltrán Garrido Juan Manuel, Benavent Martínez Luisa, Bermúdez Bellido Maribel, Blasco Asensi David, Blasco Blasco Susana, Borrás Moliner $\mathrm{M}^{\mathrm{a}}$ José, Bou Monterde Ricardo, Buch García María José, Cabrera Quintero Alberto, Calatayud Sánchez M. Ángeles, Calderón Martínez Lola, Calero Ródenas Ana, Calle Barreto Jaime Danilo, Campello Payá Carmen, Carbonell Franco Empar, Carmona Martí Esther, Castellanos Martínez Maite, Chover Lara José Luis, Cilleruelo Hernández Soledad, Corrales Fernández María José, Cremades Bernabeu Israel, Diestro Contreras Aurelio, Doménech Alonso Elena, Donate López Carolina, Encarnación Armengol Amparo, Escrivá Pons Carmen, Fernández García Carmen, Fernández Martínez Sergio, Ferrándiz Espí Encarna, Flores Muñoz Magdalena, Fuster Camarena Nieves, Fúster Pérez Marina, García Abad Isidro, García Gasulla Joan, García González María Fran- cisca, García González Concepción, García López de Meneses Ma Victoria, Gea Velazques de Castro María Teresa, Gil Aparicio María Dolores, Gil Carbonell María José, Giner Ferrando Empar, Giner Ortín Mar, Girbes Llopis José Antonio, Gombau Baldrich Carmina, Gómez Pajares Fernando, Gomis Viñedo Rosa, González Martínez Rosa, González Monte Carmen, González Torga Antonio, Guardiola Vilarroig Sandra, Guasch Aguilar Andrés, Guiral Rodrigo Silvia, Hernández Galve Alicia, Hernández Vidal Pedro, Herrero Carot Concha, Holgado Hernández María Teresa, Holguín Gómez Rita, Huertas Zarco Isabel, Ibáñez Gómez Inmaculada, Jorques Aracil Guillermo, Julve Negro Irene, Justamante Bellod Elia, Larrea González Rosa María, Leutscher Vasen Edith, Llacer Fortea Inmaculada, López Lozano José María, López Madurga Eva, López Poma Juan Carlos, Manríquez Blázquez Rosa Romana, Marco Aledo Rosario, Marco Cabero Vicente, Marín Sanchos Celia, Martín Rico Patricia, Martín-Aragón González Eva, Martínez Mantecón Manuel, Martínez Ruiz Josefa, Martín-Sierra Balibrea Miguel, Mayordomo Fernández Carlos, Melero García María Mercedes, Merino Egea Cayetano, Micó Esparza José Luis, Momparler Carrasco Pilar, Montesinos Butrón Pilar, Montesinos Carratalá María Ángeles, Morera Sobà Vicent, Mulet Falcó Francisco, Navarro Gracia Juan Francisco, Navarro Tarazada Natalia, Noguera Jiménez Emilia, Oltra Alcaraz Carlos Emilio, Ortí Lucas Rafael Manuel, Orts Canchis María José, Pascual Moltó Mercedes, Peñuelas Ruiz José Amancio, Pérez Pérez Elvira, Pérez Torregrosa Gerardo R, Pinazo Murria María, Puchades Oliver Carla, Quirós Morato Carmen, Rebollo Sachetich Begoña, Repulles Giménez Lucía, Requena Puche Juana, Rey Talens Milagros, Rigo Medrano María Vicente, Risent García Patricia, Roda Ramón Jorge, Rodrigo Bartual Vicente, Roig Sena Francisco. Javier, Romeu García Ángeles, Rosa Miguel Álvaro, Rufino Bey Ricardo, Sabater Pons Antonio, Safont Adsuara Lourdes, Sahuquillo Arce José 
Miguel, Saiz Sánchez Carmen, Salazar Cifre Antonio, Salinas Martínez Josefina, Sánchez Carratalá Encarna, Sánchez Payá José, Sarrión Auñón Antonio, Saurí Gaspar Neus, Segarra Castelló Leopoldo, Sentrandreu Mahiques Miquel, Silvestre García Assumpta, Silvestre Silvestre Esther, Soriano Llinares Laura, Taberner Cuasp Rosa Encarnación, Tabernero Escrig Teresa, Tenías Burillo José María, Valls Jiménez Victoria, Verdú Pérez Mercedes, Vicens Monerris Yolanda, Vicente Martínez Oscar, Vicente Martínez Encarnación, Vila Roig Bernardo, Zanón Viguer Vicente.

\section{BIBLIOGRAFÍA}

1. World Health Organization. Human infection with new influenza A (H1N1) virus: clinical observations from Mexico and other affected countries, May 2009 . Wkly Epidemiol Rec. 2009 ; 84(21):185-9.

2. Centers for Disease Control and Prevention. Update: Swine Influenza A (H1N1) Infections - California and Texas, April 2009. Morb Mortal Wkly Rep. 2009; 58(16):435-7.

3. Garten RJ, Davis CT, Russell CA, et al. Antigenic and genetic characteristics of swine-origin 2009 $\mathrm{A}(\mathrm{H} 1 \mathrm{~N} 1)$ influenza viruses circulating in humans. Science. 2009;325:197-201.

4. Baker M, Kelly H, Wilson N. Pandemic H1N1 influenza lessons from the southern hemisphere. Euro Surveill. 2009;14.

5. Wilson N, Baker MG. The emerging influenza pandemic: estimating the case fatality ratio. Euro Surveill. $2009 ; 14$.

6. OMS. Pandemic (H1N1) 2009 - update 80. Weekly update (Virological surveillance data). 2009.

7. Belongia EA, Kieke BA, Donahue JG, et al. Effectiveness of inactivated influenza vaccines varied substantially with antigenic match from the 20042005 season to the 2006-2007 season. J Infect Dis. 2009; 199:159-167.

8. Serum cross-reactive antibody response to a novel influenza A (H1N1) virus after vaccination with seasonal influenza vaccine. MMWR Morb Mortal Wkly Rep. 2009;58:521-524.

9. Garcia-Garcia L, Valdespino-Gomez JL, LazcanoPonce E, et al. Partial protection of seasonal trivalent inactivated vaccine against novel pandemic influenza A/H1N1 2009: case-control study in Mexico City. BMJ. 2009;339:b3928.

10. Effectiveness of 2008-09 trivalent influenza vaccine against 2009 pandemic influenza A (H1N1) United States, May-June 2009. Morb Mortal Wkly Rep. 2009;58:1241-1245.

11. Kelly H, Grant K. Interim analysis of pandemic influenza (H1N1) 2009 in Australia: surveillance trends, age of infection and effectiveness of seasonal vaccination. Euro Surveill. 2009;14.

12. Janjua NZ, Skowronski DM, Hottes TS, et al. Seasonal vaccine and H1N1. Selection bias explains seasonal vaccine's protection. BMJ. 2009;339: b4972.

13. Puig-Barbera J, Diez-Domingo J, Perez Hoyos S, Belenguer Varea A, Gonzalez Vidal D. Effectiveness of the MF59-adjuvanted influenza vaccine in preventing emergency admissions for pneumonia in the elderly over 64 years of age. Vaccine. 2004;23:283-289.

14. Farrington CP. Estimation of vaccine effectiveness using the screening method. Int J Epidemiol 1993; 22:742-6.

15. Orenstein WA, Bernier RH, Dondero TJ. Field evaluation of vaccine efficacy. Bull WHO 1985; 63:1055-1068.

16. Gross P, Russo C, Teplitzky M et al. Time peak serum antibody response to Influenza vaccine in the elderly. Clin, Diagn Lab Inmunol.1996; 361362.

17. Hancock K, Vic Veguilla, Xiuhua et al. CrossReactive antibody responses to the 2009 pandemic H1N1 Influenza virus. N Engl J Med 2009; 361: 1945-52.

18. Ikonen N, Strengell M Kinnunen et al. High frequency of cross- reacting antibodies against 2009 pandemic influenza $A(\mathrm{H} 1 \mathrm{~N} 1)$ virus among the elderly in Finland. Euro Surveill. 2010; 15 (5).

19. Goodwin K, Viboud C and Simonsen L. Antibody response to influenza vaccination in the elderly: A quantitative review. Vaccine 2006; 24:1159-1169. 